\title{
BMJ Open ADding negative pRESSure to improve healING (the DRESSING trial): a RCT protocol
}

\author{
Brigid M Gillespie, ${ }^{1}$ Joan Webster, ${ }^{2}$ David Ellwood, ${ }^{3}$ Helen Stapleton, ${ }^{4}$ \\ Jennifer A Whitty, ${ }^{5}$ Lukman Thalib, ${ }^{6}$ Nicky Cullum, ${ }^{7}$ Kassam Mahomed, ${ }^{8}$ \\ Wendy Chaboyer ${ }^{9}$
}

To cite: Gillespie BM, Webster J, Ellwood D, et al. ADding negative $\mathrm{pRESS}$ ure to improve healING (the DRESSING trial): a RCT protocol. BMJ Open 2016:6:e010287. doi:10.1136/bmjopen-2015010287

- Prepublication history for this paper is available online. To view these files please visit the journal online (http://dx.doi.org/10.1136/ bmjopen-2015-010287).

Received 18 October 2015 Revised 19 November 2015 Accepted 23 December 2015

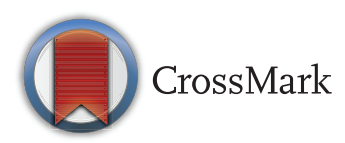

For numbered affiliations see end of article.

Correspondence to Professor Brigid M Gillespie; b.gillespie@griffith.edu.au

\section{ABSTRACT}

Introduction: Obese women are more likely to develop a surgical site infection (SSI) following caesarean section (CS) than non-obese women. Negative pressure wound therapy (NPWT) is increasingly being used to reduce SSI with limited evidence for its effectiveness.

Objectives: To determine the clinical and costeffectiveness of using NPWT in obese women having elective and semiurgent CS.

Methods and analysis: A multisite, superiority parallel pragmatic randomised controlled trial with an economic evaluation. Women with a body mass index (BMI) of $\geq 30$, booked for elective and semiurgent CS at 4 Australian acute care hospitals will be targeted. A total of 2090 women will be enrolled. A centralised randomisation service will be used with participants block randomised to either NPWT or standard surgical dressings in a 1:1 ratio, stratified by hospital. The primary outcome is SSI; secondary outcomes include type of SSI, length of stay, readmission, wound complications and health-related quality of life. Economic outcomes include direct healthcare costs and cost-effectiveness, which will be evaluated using incremental cost per quality-adjusted life year gained. Data will be collected at baseline, and participants followed up on the second postoperative day and weekly from the day of surgery for 4 weeks. Outcome assessors will be masked to allocation. The primary statistical analysis will be based on intention-to-treat.

Ethics and dissemination: Ethics approval has been obtained from the ethics committees of the participating hospitals and universities. The findings of the trial will be disseminated through peer-reviewed journals, national and international conference presentations.

Trial registration number: ACTRN12615000286549; Pre-results.

\section{BACKGROUND AND RATIONALE}

Between 187 and 281 million surgical procedures are performed around the world each year, or 1 for every 25 people. ${ }^{1}$ Surgical site infections (SSIs) are defined as infections occurring up to 30 days after surgery that affect the incision, deep tissue at the operation site or involve the organs or body spaces. ${ }^{2}$ Of concern is that SSIs occur in up to $30 \%$ of all surgical procedures, and are the third most commonly reported hospital acquired infection in many countries. ${ }^{3-5}$ SSIs have many negative effects including increasing the risk of death, prolonging hospitalisation and increasing costs. ${ }^{4}$

Obesity is an independent predictor of SSI. ${ }^{6}{ }^{7}$ Obese pregnant women are twice as likely to have a caesarean section (CS) than non-obese women. ${ }^{8}{ }^{9}$ Postoperative infection is a potential complication of all surgeries including CS; however, overweight and obese women are three times more likely to develop a SSI. ${ }^{10}$ SSI extends hospital length of stay (HLOS) by up to 6 days in women undergoing obstetric and gynaecological surgery and hospital readmission is more likely, increasing hospital costs by US $\$ 14000$ for each SSI. ${ }^{11}$

The use of negative pressure wound therapy in primary wounds

Negative pressure wound therapy (NPWT) is widely used, particularly in the management of wounds healing by secondary intention and for skin grafts. ${ }^{12-15}$ However, NPWT is increasingly being applied prophylactically to closed surgical wounds in high-risk populations to reduce the incidence of SSI. This use of prophylactic NPWT is generally applied to wounds perceived as being at high risk of SSI, for example, CS incisions in obese women. ${ }^{14} 15$ A recent Cochrane review concluded that evidence for the clinical effectiveness of prophylactic NPWT in reducing SSI and wound dehiscence is inconclusive. $^{16}$

Two further randomised controlled trials (RCTs) of NPWT have subsequently been 
published; both were feasibility studies. ${ }^{17} 18$ One of these trials examined the use of NPWT in 70 patients undergoing primary hip replacement. ${ }^{18}$ The other pilot study ${ }^{17}$ recruited 92 women undergoing elective CS and has demonstrated that a definitive trial is feasible. Neither of these trials was powered to find an effect, so the benefits or harms of NPWT for prophylactic use remain unclear.

Thus, a RCT to evaluate the clinical and costeffectiveness of NPWT in obese women undergoing CS is timely and responds to the imperative to provide much-needed evidence to guide practice in a rapidly developing and costly area of healthcare.

\section{Primary objective}

To compare the effects of prophylactic NPWT and standard surgical dressings on the incidence of SSI in obese women undergoing $\mathrm{CS}$.

\section{Secondary objectives}

To compare: (1) the incidence of superficial, deep, organ/space SSI; (2) the number of dressing changes; (3) the number and type of wound complications (ie, dehiscence, haematoma, seroma, adverse events (AEs)); (4) the number of hospital readmissions; (5) HLOS (days); (6) health-related quality of life (QoL); and (7) direct healthcare costs in obese women undergoing CS who receive prophylactic NPWT with women who receive standard surgical dressings.

\section{Study design}

Multicentre, parallel group, pragmatic, randomised controlled superiority trial.

\section{METHODS}

\section{Study setting and population}

Four acute care public hospitals offering obstetric services in Queensland, Australia, have agreed to participate. Across the four sites, the number of births ranges from 2400 to 10000 per annum. The study population will consist of 2090 women with a BMI of $\geq 30$, undergoing either elective or semiurgent CS.

\section{Eligibility criteria}

According to national and international guidelines, CS urgency is based on these four categories: (1) lifethreatening to woman or fetus; (2) maternal or fetal compromise, not life-threatening; (3) needing earlier CS than planned without maternal or fetal compromise; and (4) a scheduled time acceptable to the woman and CS team. ${ }^{19} 20$

Inclusion criteria:

1. Women booked for elective CS surgery (category 4);

2. Women whose condition changes to require a semiurgent CS (categories 2-3);

3. Recorded pre-pregnancy BMI of $\geq 30$ at the first antenatal visit; and
4. Able to provide written informed consent.

Exclusion criteria:

1. Women who require an urgent CS (category 1) at any point;

2. Existing infection after admission to hospital in labour/immediately prior to CS;

3. Previous participation in this trial; and

4. Unable to speak or understand English, with no interpreter available.

\section{Interventions}

While this will be a pragmatic trial, a checklist based on published current clinical practice guidelines and Queensland Maternity and Neonatal Guidelines will be used to standardise the CS surgical procedure. The participating obstetrician's clinical judgement may produce slight variation in practice in the type of wound closure (ie, closure of facial layer as well as rectus muscle); selection of suture materials (ie, staples vs subcuticular absorbable suture for skin); and standard dressing preference (eg, semipermeable vs hydrocolloid). There is no evidence to suggest that the aforementioned minor variations in clinical practice increase the risk of SSI.

\section{Intervention}

At the completion of skin closure, women randomly allocated to the NPWT arm of the trial will receive a PICO (Smith \& Nephew, Hull, UK) dressing applied by the obstetrician under sterile conditions. The PICO product was chosen because it is lightweight and disposable, significantly cheaper than other options, and has performed well in a pilot study. ${ }^{17}$ It comprises a small, discrete pump, powered by two AA-lithium batteries with a highly absorbent dressing that holds the wound exudate away from the skin, thus negating the need for a bulky canister. The polyurethane foam dressing will be secured over the incision by the application of an adhesive drape. A tube is embedded into the foam, and continuous negative pressure of $80 \mathrm{~mm} \mathrm{Hg}$ will be applied to the dressing.

\section{Control}

Women in the control arm will have a standard dressing based on the obstetrician's usual preference, applied according to the manufacturer's recommendations after skin closure. In both groups, we anticipate the dressing will remain in situ for 4- 5 days, unless it becomes soiled or dislodged, in which case a new dressing of the same type will be applied. Given the pragmatic nature of this trial, the number of days dressings are left in situ and the number of dressings used will be recorded. To ensure consistency, clinicians providing care to the target population at each of the sites will receive trialspecific education (NPWT and standard). The research assistants (RAs) who will also receive trial-specific training will be available to clinical staff during business hours to provide ongoing training and support about correct use of the dressings, as well as monitoring 
dressing changes and completing documentation daily to assess protocol compliance and outcomes. If our monitoring shows variation from the proposed protocol, this variation will be used as a factor in the analyses.

\section{Outcome measures}

The primary outcome is the incidence of an SSI in the CS wound at any time up to 28 days after surgery.

The following secondary outcomes will be assessed: depth of SSI, that is, superficial, deep or organ/body space ${ }^{3}$; number of dressing changes; presence and number of wound complications (ie, dehiscence, haematoma, seroma, blisters).

Other secondary outcomes: HLOS will be measured in days; number of hospital readmissions will be measured within 4 weeks from the day of surgery. The secondary outcome, health-related QoL will be assessed using the Short Form (SF)-12v2 (acute 1-week recall). The SF-12v2 will be administered at baseline (ie, recruitment at $>36$ / 40 weeks in women having elective CS, or on the day of CS for women undergoing semiurgent CS), and via telephone interview weekly for four consecutive weeks after surgery. Direct healthcare costs will be included as part of an economic evaluation.

\section{Participant timeline and trial duration}

Participants will be enrolled in the study for 28 days from the day of surgery (Table 1 ). The project will take up to 5 years to complete, with recruitment and data collection expected to occur over 3.5 years. Participants will exit the trial when they: withdraw consent; have been in the trial for 28 days postrandomisation; are lost to follow-up; die; or for another reason have to exit based on the clinical judgement of the attending healthcare professional.

\section{Sample size}

The sample size was calculated based on the primary outcome, SSI. Based on other related published studies, ${ }^{21}{ }^{22}$ we conservatively estimate the baseline SSI incidence in obese CS women to be $15 \%$. Following discussions with infectious disease experts and obstetricians, we have accepted an absolute difference between groups of $5 \%$ to be clinically important. Therefore, to achieve over $90 \%$ power to test the superiority of SSI incidence between groups, 950 women per group will be required (Power Analysis \& Sample Size system (PASS, V.12), NCSS). To allow for attrition, a further $10 \%$ $(n=95)$ will be recruited to each group for a total sample of 2090 (1045/group). We anticipate recruiting approximately 600 women per year, thus recruitment should be completed in about 3.5 years.

\section{Recruitment of participants}

We will use a staged approach to recruitment, starting at one site to test procedures, prior to starting at the other sites. All obese women will be given an information brochure informing them of the trial during their routine antenatal visit at 36 weeks. This strategy will facilitate further discussion and assist with consent processes in labour in the event that a semiurgent CS is required. Women booked for an elective CS will be given the opportunity to provide written consent during their antenatal visit (36/40 weeks). On the day of surgery, women undergoing elective CS will be screened to ensure they continue to meet the inclusion criteria; those who have not yet provided written consent will be invited to do so. Women undergoing semiurgent CS will be consented on the day of surgery. Figure 1 shows anticipated participant flow through the study.

\section{Implementation of sequence generation and allocation concealment}

Women booked for elective CS will not be randomised at recruitment as the long time lag between randomisation and receiving the intervention which could lead to poor adherence to allocation and loss to follow-up. All women who continue to meet the eligibility requirements will be randomised using a centralised, independent, web-based randomisation system. The RA will randomise participants in the operating room at the start of their CS procedure and advise the operating obstetrician and nursing staff of the allocated treatment as close to the end of the procedure as possible in order to minimise performance bias. The allocation sequence will be used to ensure allocation concealment. To reduce predictability of a random sequence, randomly varying block sizes of four, six and eight will be used.

\section{Blinding}

This pragmatic trial tests a clinical intervention that is not amenable to protection against performance bias through the blinding of participants, clinical staff or data collectors.

To minimise the potential for outcome detection bias, an expert clinician, blinded to group allocation, will assess the data to determine the primary outcome. The trial statistician and coordinating principal investigator (PI) will also be blinded. It is unlikely that entries to the medical records and hospital databases will be falsified; thus, lack of blinding for these outcomes should not affect the data integrity. Subjective outcomes (wound complications, QoL) are reported by patients or observed by RAs, who cannot be blinded because they need to check the dressings and document participants' responses.

Performance bias is a consideration when clinical staff cannot be blinded. To assess the risk of performance bias, a standardised set of questions will be used to document the number of dressing changes and/or protocol violations occurring during the hospital stay, and following discharge. We considered using dressings where tubing was attached to a suction apparatus in both groups; however, participants, staff and data collectors would almost certainly be aware if suction was activated 


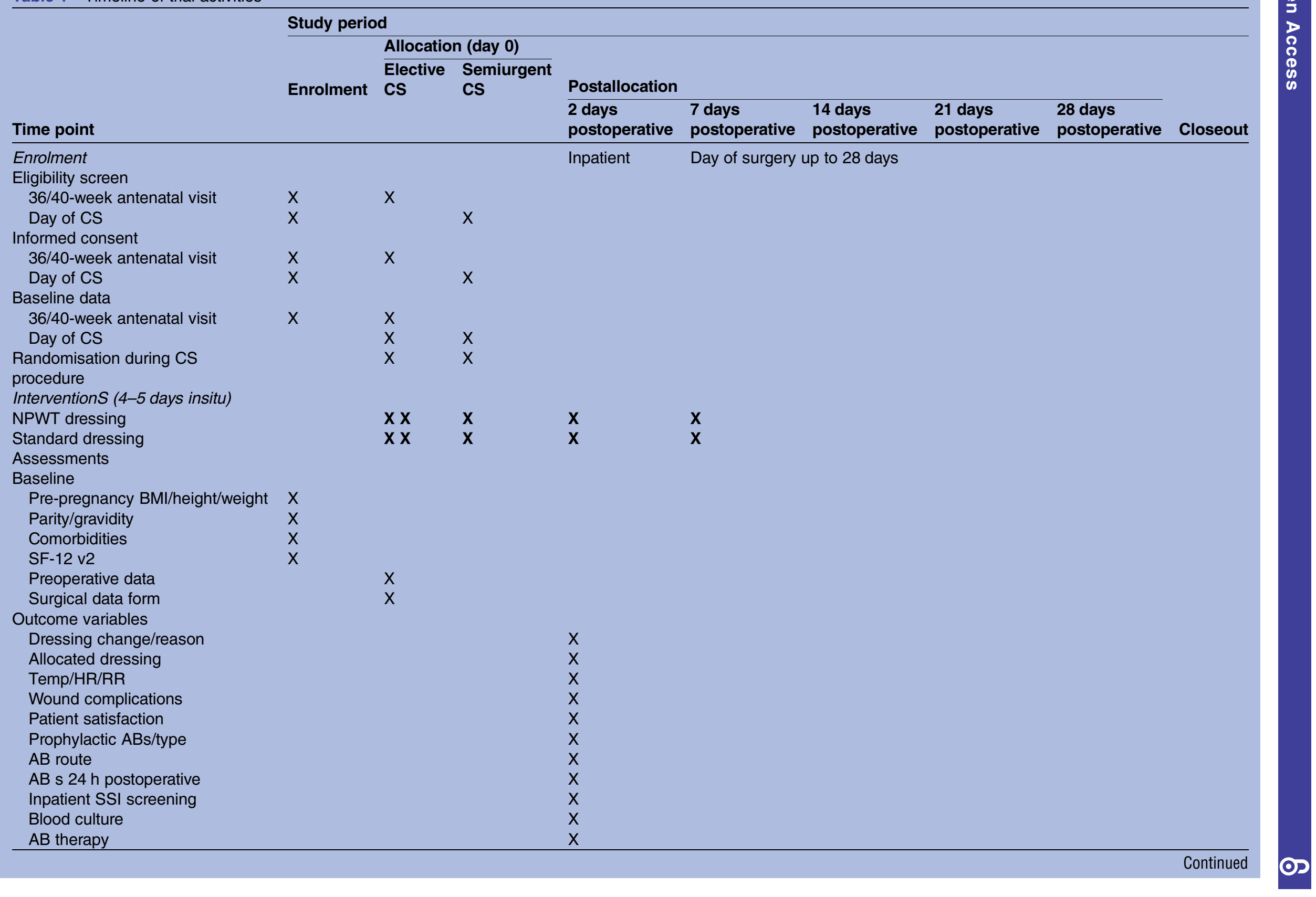




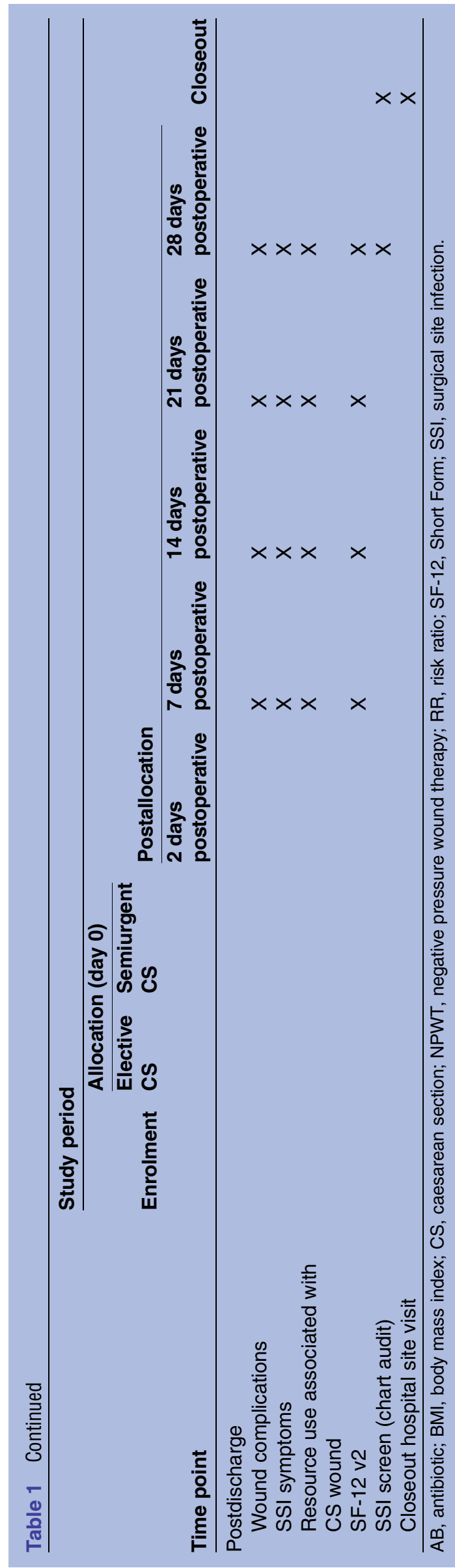

and we decided it was most important to measure 'realworld' effects and conduct a pragmatic trial.

\section{Data collection}

The RAs will collect SSI-related data on day 2 (postsurgery) using a structured form. In Queensland, Australia, the Centre for Healthcare Related Infection Surveillance and Prevention provides guidelines identifying SSI signs and symptoms (ie, redness, swelling, pain/tenderness, dehiscence, watery or purulent discharge), both during hospitalisation and after hospital discharge. Data which will be collected from a variety of sources including chart audit, direct observation and patient self-report both during hospitalisation and after discharge. RAs will record dressings used and antibiotic medication. Data collection will occur on weekdays (Monday to Friday). Clinical outcome data will be collected by the RAs retrospectively though telephone interviews and by accessing participants' medical records.

After hospital discharge, all women will be telephoned weekly (from their day of surgery) and outcomes assessed using a series of questions, which have been used successfully in other research in this area. ${ }^{17} 1822$ Seven-day recall of SSI symptoms and related resource use including health professional visits (eg, consultations with general practitioners (GPs)) was demonstrated as being feasible in a recently published pilot trial ${ }^{17}$ and will allow accurate SF-12v2 and costing data to be collected.

RAs will collect and directly enter data while in the clinical areas, using portable computers with a purposebuilt database and form-based interface (ie, Research Electronic Data Capture (REDCap) database). Clinical characteristics such as age, comorbidities and other risk factors for SSI, such as nicotine use and length of operation, will be collected at baseline. Recruitment and data collection will be monitored by the clinical trial coordinator (CTC) weekly and monthly reports will be presented to the study investigators. While there is a potential for loss to follow-up, our pilot study has demonstrated retention rates of $>85 \%$. ${ }^{17}$ We will also use standard procedures, such as recording alternative phone and email contacts for participants and GPs, in order to assist with tracing women who may have moved house/changed internet providers, etc.

\section{Ascertainment of the primary outcome}

SSI-related data will be given to the two blinded expert clinicians, who will compare the data against criteria defined by the Centres for Disease Control and Prevention Guideline for Prevention of $\mathrm{SSI}^{3}$ to decide if a SSI exists. We will use decision rules to ascertain the primary outcome. If the two blinded expert clinicians disagree on whether the patient has sustained a SSI within the 28-day postoperative period, we will use the following combinations with regard to wound infection: yes/no, yes/unsure, no/ unsure: 
Figure 1 Anticipated participant flow through study.

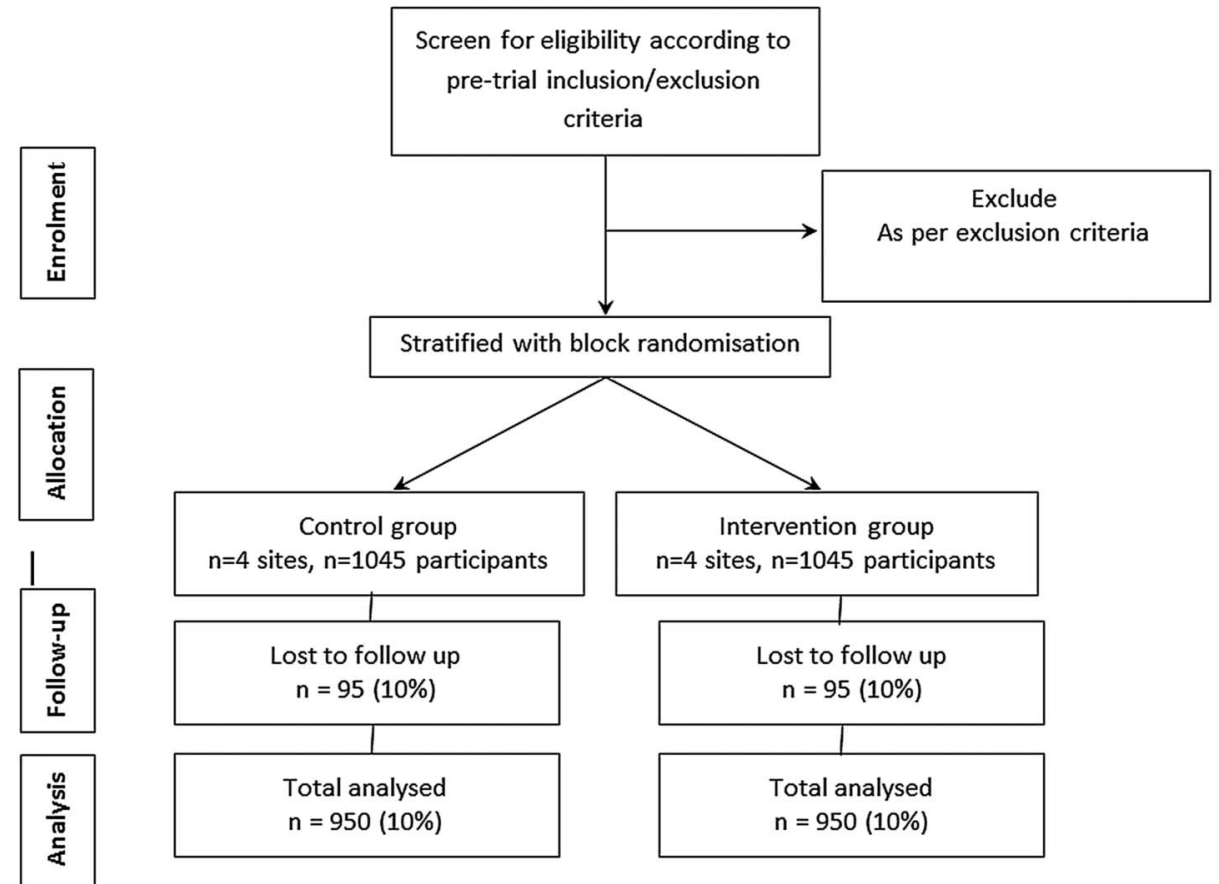

1. If the two assessors say: 'yes/unsure' then we will say the patient has a SSI, using the data provided by the assessor who said 'yes'.

2. If the two assessors say: 'no/unsure' then we will say the patient does not have a SSI.

3. If the two assessors say: 'unsure/unsure' then we will say the patient does not have a SSI.

4. If the two assessors say: 'yes/no' then the third assessor will be consulted and will decide if there is a SSI or not. The third assessor's decision will be final. If $\mathrm{s} /$ he is unsure whether the patient has a SSI or not, then the patient will be considered not to have a SSI.

\section{Training, outcome assessment and treatment fidelity}

Adhering to Good Clinical Practice (GCP) recommendations, ${ }^{23}$ this multisite study will have an experienced CTC coordinating the RA training, and site and data monitoring. Trial-specific RA training to assess the patient's incision/dressing site will be provided by a tissue viability nurse. Additional training in the use of the PICO NPWT dressing product will be provided by a Smith \& Nephew clinical nurse educator with specialist knowledge but with no role in the design, analysis or reporting of the study. Involving a product specialist in the training of the RAs and clinicians who will be using the PICO product ensures that all end-users receive comprehensive and consistent information relative to the appropriate use and management of the study intervention. The RAs will undergo group and individual onsite training to ensure consistency across the four sites. Areas covered will include recruitment and data collection processes, use of the central randomisation service and database.

Consistent with GCP recommendations, ${ }^{23}$ a standardised operating procedure manual has been developed to provide more specific detail on the protocol, plans for dealing with intervention fidelity issues, and monitoring the delivery and receipt of the intervention. Assessment of treatment fidelity will focus on type of dressing used, duration of use and number of dressing changes. While this is a pragmatic trial, obtaining information on intervention fidelity may help to explain study results. All members of the research team, including RAs, will be provided with training, a procedure manual and a DVD detailing the NPWT dressing application to ensure protocol consistency. A trial-specific training programme and ongoing education sessions targeting obstetricians, operating room staff, midwives and nurses will also be implemented at each site.

\section{Withdrawal}

If a participant decides to withdraw from the trial after consent is given, any existing data obtained during the trial will be retained and no further follow-up data collected. A withdrawal form will be completed and reasons for withdrawal noted. Patients who withdraw from randomised treatment prior to randomisation will be left in the study and reported in the flow chart as 'not receiving randomised intervention'. Patients who withdraw after they have received the intervention (ie, after dressing application) will also be included and reported in the flow chart as 'receiving randomised treatment'.

\section{Data management}

All data will be managed using a centralised REDCap (Nashville, Tennessee, USA) database repository, hosted on a dedicated secure server within the Clinical Trials Unit at Griffith University. The trial statistician will not have access to the database to ensure he remains 
blinded to group allocation. This database enables different levels of data access, allowing researcher control over who sees the files and what they can do with them. Data access will therefore be restricted and all data password protected. Each site RA will have a level of access to the database specific to their site and position, and will use a password-protected tablet computer for data entry into REDCap.

An electronic case report form (eCRF) will be developed in collaboration with the trial statistician, coordinating PI and CTC. The eCRF will be hosted on the REDCap database within the Griffith University's Clinical Trials Unit. Source data will be entered by the site RAs, who will receive trial-specific training in the use of the eCRF. All entered data will be directly exported into the IBM Statistical Package for the Social Sciences (SPSS V.22.0, New York, USA) for analysis.

\section{Identifiable data}

For the purposes of statistical analysis, data will be anonymised and collated by the CTC and uploaded into SPSS. Identifiable data will be entered on the eCRF to enable follow-up. These data will be de-identified when transferred to the statistical database. Participants will be identified on the statistical database using a unique code and hospital site initials.

\section{Statistical analysis}

\section{Primary analyses}

Prior to analysis, a rigorous process of data cleaning to check outlying figures, missing and implausible data against source data will be undertaken. Baseline characteristics of the patients in each arm of the trial will be calculated using descriptive statistics. We will employ an intention-to-treat approach for the primary analysis with the population defined as all trial participants who completed the baseline assessment and underwent surgery and received the intervention. AEs will be analysed and reported on a per protocol analysis. The incidence of SSI per 1000 patients between groups will be compared. Risk ratios (RR), 95\% CIs and $\mathrm{p}$ values assuming a $5 \%$ significance level will be presented. For the primary outcome, the number needed to treat and absolute risk reduction will be calculated from the RR. While we do not anticipate differences between groups in terms of known or unknown prognostic factors due to randomisation, adjusted analyses using multivariate logistic regression models will be used if any difference in prognostic variables is detected. Despite every effort to minimise missing values it is possible that some may occur. We will evaluate the utility of empirical imputation methods in such cases and only impute them if the explanatory power of the empirical imputation models is robust. To assess the representativeness of the sample, we will compare the characteristics of the women in our sample with secondary data available at state and national levels.

\section{Secondary analyses}

Secondary end points will be compared between groups using statistical methods appropriate to the distribution of measures. A random sample of $5 \%$ of the data will be rechecked for accuracy against source data. RRs with 95\% CI will be calculated for clinical outcome data. Analysis will be performed by one of the study investigators (blinded to group allocation), trained in biostatistics.

\section{Economic evaluation}

Direct costs to the healthcare system will be obtained from patient records/hospital cost centres (during hospital admission) and self-reported by women (weekly for 4 weeks post surgery). Resources costed during admission will include dressings and related wound management products, medications related to SSI (ie, use of antibiotics), and resources used to manage any adverse effects of the dressing. Use of dressings, medications and health professional appointments related to wound management will be recorded at weekly intervals for 4 weeks following surgery. Direct costs will be assigned using standard costing sources (eg, Medicare Benefits and Pharmaceutical Benefits Schedules; Independent Hospital Pricing Authority). The Australian-refined diagnostic-related groups will be used to indicate the costs associated with each hospital admission, adjusted for HLOS.

\section{Cost-effectiveness analysis}

A within-trial economic evaluation will be undertaken from the health system perspective to compare the costs and effects of NPWT, relative to the standard dressing. Parametric (eg, analysis of covariance) or nonparametric bootstrapping techniques ${ }^{24}$ will be employed to compare the mean difference in the total costs between groups. The cost-effectiveness analysis will be undertaken based on the primary outcome measure (SSI). Additionally, a cost-utility analysis will be performed using the quality-adjusted life year (QALY) as the outcome measure. The QALY gain associated with NPWT will be estimated based on SF-12v2 utility weights. $^{24}$ Uncertainty around the incremental costeffectiveness ratios will be tested using one-way sensitivity analysis and non-parametric bootstrapping methods. ${ }^{24}$ The cost-effectiveness estimates will inform recommendations on adopting NPWT dressings for CS surgical wounds in clinical practice.

\section{Safety and data monitoring}

An $\mathrm{AE}$ is defined as an untoward medical occurrence experienced by the participant, whether or not considered treatment related. ${ }^{25}$ In this trial, AE will be classified as non-serious (infection, pain, maceration, odour) and serious (dehiscence, return to theatre). Both types of $\mathrm{AE}$ would be expected to occur equally in both treatment groups. Patients will be monitored for potential AEs, serious and non-serious. All AEs reported will be 
assessed to determine whether further diagnostic investigation or treatment is warranted. If an AE occurs, appropriate treatment will be given. Monitoring and reporting of suspected unexpected adverse reactions (SUSARs) will be performed by the site PI and the research team. All SUSARs will be recorded on a dedicated eCRF. Serious AEs associated with the intervention are considered unlikely, although if any are reported the relevant Human Research Ethics Committees will be notified, with appropriate notification of the Therapeutic Goods Administration (TGA) as required.

The results of the trial will be reviewed every 6 months by an independent Data Safety and Monitoring Committee (DSMC) including an obstetrician, a statistician and a tissue viability nurse, all of whom will be independent of the study. The DSMC has the ability to terminate the trial prematurely if there is unacceptable harm associated with the treatment. ${ }^{26}$ If requested by the DSMC, an interim analysis will be performed by a statistician (independent of the DSMC), blinded for the treatment allocation.

\section{Auditing}

Data monitoring of this trial will ensure compliance with GCP. $^{23}$ The participating sites will provide access to all trial-related source data/documents and reports for the purposes of monitoring, auditing and inspection by local authorities. The CTC will undertake monitoring in relation to the accuracy of the case report data collected by the site RAs. During the trial, the CTC will use source data to verify the data entered by the RAs into the eCRF.

\section{Ethics and dissemination}

This trial has been approved by the relevant hospital ethics boards and the respective universities where the PIs are employed. This study will be conducted in accordance with the principles of the Declaration of Helsinki (1996), and the Australian National Health and Medical Research Council (NHMRC) National Statement. ${ }^{27}$

Dissemination strategies will include knowledge translation events involving opinion leaders and stakeholders. Findings will be presented at local hospital and other fora and a press release will be prepared. A succinct non-technical paper discussing the relevance of findings and application to practice, and recommendations for future research, will be prepared and disseminated to the colleges representing the relevant health professional groups. Abstracts will be submitted to major international meetings of infection control, nursing/ midwifery and medical groups. We envisage the results will be published in high-impact generalist and specialist journals. We will bring the published study to the attention of the Cochrane Wounds Group and the authors of the relevant Cochrane review to ensure early inclusion in review updates. The results will have international application and we anticipate that they will be rapidly adopted and cited within the GCP literature.

\section{DISCUSSION}

Despite a lack of rigorous evidence to support either clinical or cost-effectiveness, NPWT is increasingly being used as a prophylaxis against SSI in high-risk surgical groups such as obese women undergoing CS. To the best of our knowledge, this RCT will be the largest of its kind in this area. It has significant potential to inform practice because it assesses the clinical and cost-effectiveness of using NPWT in a patient population at high risk of incurring an SSI. A RCT design with a sufficiently large sample is time consuming and expensive to undertake, but it is nevertheless necessary to determine the efficacy of NPWT in the management of surgical incisions.

Our trial has several strengths. First, the RCT design with a robust randomisation process will ensure that any difference in outcomes between the groups is attributable to the intervention. Second, a pragmatic approach allows testing of an intervention that reflects the reality of the clinical environment relative to study population, intervention, comparator and outcomes. ${ }^{26}$ Third, an independent clinician, blinded to group allocation will assess these data to determine SSI status. Fourth, the embedding of an economic evaluation responds to the need to provide healthcare administrators and decisionmakers with meaningful cost-effectiveness data. Finally, this trial is independent of industry funding, and therefore minimises potential for a conflict of interest which may bias the results.

While we envisage that the results of this trial will provide clinicians with definitive answers around the effectiveness of NPWT in this specific patient population, undertaking this 5-year trial is not without its challenges, including meeting our recruitment targets within a 3.5-year timeframe. For each hospital site, yearly recruitment targets will need to range from 120 to 200 women. To maximise both recruitment and generalisability, we will include women who are undergoing both elective and semiurgent CS. Another major challenge is the potential for missing outcome data due to participant attrition as participants in this study will typically be busy with childcare and other commitments postoperatively. Yet in a recent pilot study, participant attrition was less than $10 \%,{ }^{17}$ which is considered acceptable. Maximising participant retention over a 4-week follow-up period will be achieved by the RAs meeting women face-to-face during pregnancy/labour and again postoperatively. These meetings will enable a relationship of trust to be developed and allow the RAs to resolve any queries promptly. It will also provide opportunities for the RAs to reconfirm women's contact details and remind them about the four weekly telephone followups. In the event that an RA is unsuccessful in their first attempt to contact women following discharge, they will try again, perhaps using their alternative contact details. Finally, during the 3.5-year data collection period, there may be clinical innovations introduced that potentially influence the trial outcomes (ie, 'history'). However, we anticipate that both groups to be equally influenced. 
Author affiliations

${ }^{1}$ NHMRC Centre for Research Excellence in Nursing, Centre for Health Practice Innovation (HPI), Menzies Health Institute Qld (MHIQ), Griffith University, Gold Coast, Queensland, Australia

${ }^{2}$ Research Centre for Clinical Nursing, Royal Brisbane and Women's Hospital and Griffith University, Nathan, Queensland, Australia

${ }^{3}$ Department of Obstetrics \& Gynaecology, Griffith University School of Medicine and Director of Maternal-Fetal Medicine, Gold Coast University Hospital, Australia

${ }^{4}$ Mater Research Institute and The University of Queensland (MRI-UQ), Brisbane, Queensland, Australia

${ }^{5}$ Department of Health Economics, Pharmacoeconomics \& Quality Use of Medicines, School of Pharmacy, University of Queensland, Australia ${ }^{6}$ Department of Health Sciences, College of Arts \& Science, Qatar University, Qatar

${ }^{7}$ School of Nursing, Midwifery \& Social Work, The University of Manchester, UK

${ }^{8}$ West Moreton Hospital and Health Service, Ipswich, Queensland, Australia ${ }^{9}$ NHMRC Centre for Research Excellence in Nursing, Menzies Health Institute Qld (MHIQ), Griffith University, Gold Coast, Queensland, Australia

Contributors BMG, JW and WC conceived of the study. NC, LT, DE, HS, JAW and KM contributed to the study design and will assist with implementation. All authors are grant holders. LT and NC provided methodological expertise in clinical trial design and LT is leading the primary statistical analysis. JAW provided expertise in the health economic analysis and will lead the economic evaluation. All authors contributed to refinement of the study protocol and approved the final manuscript.

Funding This trial is funded through an Australian National Health \& Medical Research Project Grant (APP1081026).

Disclaimer The funding source had no role in the design of this study and will not have any role during its execution, analyses, interpretation of the data, or decision to submit results.

Competing interests None declared.

Ethics approval Royal Brisbane \& Womens Hospital and Griffith University, Queensland Australia.

Provenance and peer review Not commissioned; externally peer reviewed.

Data sharing statement A summary of the results will be disseminated to the study participants on request. The authors plan to publish the main trial outcomes in a single paper. Further publications are anticipated after exploring the data in more detail. Findings will be presented at national and international conferences from early 2020.

Trial status At the time of manuscript submission, ethics (HREC/15/QRBWH/ 126) and contract approvals have been given. The investigator team are awaiting final approvals for research governance.

Open Access This is an Open Access article distributed in accordance with the Creative Commons Attribution Non Commercial (CC BY-NC 4.0) license, which permits others to distribute, remix, adapt, build upon this work noncommercially, and license their derivative works on different terms, provided the original work is properly cited and the use is non-commercial. See: http:// creativecommons.org/licenses/by-nc/4.0/

\section{REFERENCES}

1. 10 facts on safe surgery. http://www.who.int/features/factfiles/safe surgery/en/index.html

2. National Institute for Health and Care Excellence. NICE surgical site infection prevention and treatment of surgical site infection. London: RCOG Press at the Royal College of Obstetricians and Gynaecologists, 2008.
3. Mangram AJ, Horan TC, Pearson TC, et al. Guideline for prevention of surgical site infection, 1999. Hospital Infection Control Practices Advisory Committee. Infect Control Hosp Epidemiol 1999;20:250-80.

4. Gravel D, Taylor G, Ofner M, et al., Canadian Nosocomial Infection Surveillance Program. Point prevalence survey for healthcare-associated infections within Canadian adult acute-care hospitals. J Hosp Infect 2007;66:243-8.

5. Smyth ET, Mcllvenny G, Enstone JE, et al. Four country healthcare associated infection prevalence survey 2006: overview of the results. $J$ Hosp Infect 2008;69:230-48.

6. Anderson C, Talsma A. Characterizing the structure of operating room staffing using social network analysis. Nurs Res 2011;60:378-85.

7. Waisbren E, Rosen $\mathrm{H}$, Bader AM, et al. Percent body fat and prediction of surgical site infection. J Am Coll Surg 2010;210:381-9.

8. Heslehurst N, Simpson H, Ells LJ, et al. The impact of maternal BMI status on pregnancy outcomes with immediate short-term obstetric resource implications: a meta-analysis. Obes Rev 2008;9:635-83.

9. Callaway LK, Prins JB, Chang AM, et al. The prevalence and impact of overweight and obesity in an Australian obstetric population. Med $J$ Aust 2006; 184:56-9.

10. Ramachenderan J, Bradford J, McLean D. Maternal obesity and pregnancy complications: a review. Aust NZ J Obstet Gynaecol 2008;48:228-35.

11. de Lissovoy G, Fraeman $\mathrm{K}$, Hutchins $\mathrm{V}$, et al. Surgical site infection: incidence and impact on hospital utilization and treatment costs. Am $J$ Infect Control 2009;37:387-97.

12. Stannard JP, Volgas DA, Stewart R, et al. Negative pressure wound therapy after severe open fractures: a prospective randomized study. J Orthop Trauma 2009;23:552-7.

13. Llanos S, Danilla S, Barraza C, et al. Effectiveness of negative pressure closure in the integration of split thickness skin grafts: a randomized, double-masked, controlled trial. Ann Surg 2006;244:700-5.

14. Gregor S, Maegele M, Sauerland S, et al. Negative pressure wound therapy: a vacuum of evidence? Arch Surg 2008;143:189-96.

15. Kairinos N, Voogd AM, Botha PH, et al. Negative-pressure wound therapy II: negative-pressure wound therapy and increased perfusion. Just an illusion? Plast Reconstr Surg 2009;123:601-12.

16. Webster J, Scuffham P, Stankiewicz M, et al. Negative pressure wound therapy for skin grafts and surgical wounds healing by primary intention. Cochrane Database of Systematic Reviews 2014;10:CD009261. doi:10.1002/14651858.CD009261.pub3

17. Chaboyer W, Anderson V, Webster J, et al. Negative pressure wound therapy on surgical site infections in women undergoing elective caesarean sections: a Pilot RCT. Healthcare 2014;2:417-28.

18. Gillespie BM, Rickard CM, Thalib L, et al. Use of negative pressure wound dressings to prevent surgical site complications after primary hip arthroplasty: a Pilot RCT. Surg Innov 2015;22:488-95.

19. Gynaecologists (RANZCOG). Categorisation of urgency for caesarean section: College Statement. Royal Australian \& New Zealand College of Obstetricians \& Gynaecologists; 2011. https:// www.ranzcog.edu.au/college-statements-guidelines.html (accessed 20 Nov 2013).

20. Lucas D, Yentis S, Kinsella S, et al. Urgency of caesarean section: a new classification system. $J$ R Soc Med 2000;93:346-50.

21. Ahmed SR, Ellah MA, Mohamed OA, et al. Prepregnancy obesity and pregnancy outcome. Int J Health Sci (Qassim) 2009;3:203-8.

22. Webster J, Croger S, Lister C, et al. Use of face masks by non-scrubbed operating room staff: a randomized controlled trial. ANZ J Surg 2010;80:169-73.

23. Mathieu M. Good clinical practice: a question \& answer reference guide. Waltham, MA: Paraxel International Corporation, Barnett International, 2011.

24. Brazier J. The estimation of a preference-based measure of health from the SF-12. Med Care 2004;42:851-9.

25. de Vries EN, Ramrattan MA, Smorenburg SM, et al. The incidence and nature of in-hospital adverse events: a systematic review. Qual Saf Health Care 2008;17:216-23.

26. Williams HC, Burden-Teh E, Nunn AJ. What is a pragmatic clinical trial? J Invest Dermatol 2015;135:e33.

27. National Health \& Medical Reseaqrch Council. National Statement on Ethical Conduct in Human Research (2007)—Updated May 2015. https://www.nhmrc.gov.au/guidelines-publications/e72 